
\title{
R Reserach S Suare \\ Up-regulation of Rb by small activating RNA inhibits cell invasion and migration in gastric cancer cells
}

\author{
Lipan Peng \\ Shandong Provincial Hospital \\ Shujie Zeng \\ Shandong Provincial Hospital \\ Shuai Kong \\ Shandong Provincial Hospital \\ Shubo Tian \\ Shandong Provincial Hospital \\ Tao Xu \\ Shandong Provincial Hospital \\ Guangchuan Wang ( $\nabla$ guangchuanwang2015@126.com ) \\ Shandong Provincial Hospital https://orcid.org/0000-0002-6066-4795
}

Primary research

Keywords: Gastric cancer, RNA activation, small activating RNA

Posted Date: June 16th, 2020

DOI: https://doi.org/10.21203/rs.3.rs-35113/v1

License: (c) (1) This work is licensed under a Creative Commons Attribution 4.0 International License.

Read Full License 


\section{Abstract}

Gastric cancer (GC) is a lethal disease that needs further investigation. Recent studies have reported that small activating RNA (saRNA), involved in a process called RNA activation, plays an important role in the development of cancer. We designed three different saRNAs targeting the retinoblastoma $(\mathrm{Rb})$ gene promoter in GC cell lines. Upregulated Rb expression after transfection of the saRNAs was confirmed by PCR and western blotting and resulted in inhibition of GC cell proliferation, migration, and invasion compared with the control groups. There was no significant difference between the negative control saRNA and mock transfection groups. Overall, Rb is a promising novel prognostic biomarker of GC and, due to its role in metastasis, a novel therapeutic target for the clinical management of invasive and metastatic GC.

\section{Background}

Gastric cancer (GC) is the fifth most common tumor and third leading cause of cancer-related mortality in the world [1]. However, in China, GC has the highest morbidity and mortality rates of all cancers [2]. Although the incidence of GC is decreasing by nearly $2 \%$ per year worldwide, the overall treatment effect has not fundamentally improved due to population growth [3]. The highly invasive behavior of GCs often leads to recurrence and metastasis, which are the main causes of death in patients [4]. GC-related genes, including oncogenes and tumor suppressor genes, are closely related to the malignant biological behavior of GC [5]. Therefore, in-depth investigation of the mechanisms of GC-related genes can provide the necessary theoretical basis for identifying new targets for GC treatment.

Small activating RNAs (saRNAs) act mainly on tumor suppressor genes, including those that negatively regulate the cell cycle, promote cell death, and inhibit tumor invasion and metastasis [6]. saRNAs upregulate the expression of such genes to promote tumor cell differentiation, increase apoptosis, and inhibit tumor cells [7]. In the process of RNA activation (RNAa), the expression of saRNA target genes is upregulated after the saRNA binds to a specific region of the promoter region or against the recognition of a tumor suppressor gene by non-coding small RNAs (microRNAs) [8]. In addition, saRNAs promote post-transcriptional upregulation of target genes via different pathways [9]. Compared with the traditional methods of increasing target gene expression, RNAa has unique advantages involving simple operation, low cost, very specific activation, and physiological regulation [10]. Furthermore, its effects persist in the cell but can be terminated by stopping the drug, and the activation effect is easily controlled.

At present, the mechanism of RNAa is unclear, but different studies have shown that saRNAs activate target genes at the transcriptional level by binding to certain regions of the promoter or to the antisense transcript of the target gene $[11,12]$. Because saRNAs act primarily on oncogenes and have achieved important clinical results, we believe that saRNAs targeting tumor suppressor genes may play an important role in the treatment of GC.

\section{Materials And Methods}




\section{Cell lines and culture}

Authenticated GC cell lines, SGC-7901 and MKN-28, were obtained from the American Type Culture Collection (Manassas, VA, USA). All cell lines were cultured in Dulbecco's modified Eagle medium (Gibco, Rockville, MD, USA) supplemented with $10 \%$ fetal bovine serum (Gibco) and $1 \%$ penicillin/streptomycin under a humidified atmosphere containing $5 \% \mathrm{CO}_{2}$ at $37^{\circ} \mathrm{C}$.

\section{Cell transfection}

SGC-7901 and MKN-28 cells $\left(2 \times 10^{5} / 6\right.$-well plate) were cultured to $50 \%-70 \%$ confluence. Lipofectamine 2000 (Thermo Fisher Scientific, Waltham, MA, USA) was used to transfect the cells with $50 \mathrm{nM}$ saRNA targeting retinoblastoma $(\mathrm{Rb})$ or negative control saRNA (dsControl). The mock control was treated with Lipofectamine 2000 alone.

\section{RNA extraction and quantitative reverse-transcription PCR}

Total RNA from tissues or cells was extracted using TRIzol reagent (Invitrogen, Carlsbad, CA, USA) and was reverse transcribed using the RT-PCR kit (Tiangen, Beijing, China) according to the manufacturer's instructions. Quantitative PCR was conducted using the SYBR Green Premix reagent (Tiangen) and ABI Prism 7000 Fluorescent Quantitative PCR System (Applied Biosystems, Foster City, CA, USA) according to the manufacturer's instructions. The sequences of the primers used in this study are as follows: forward, 5'- CTC TCG TCA GGC TTG AGT TTG-3' and reverse, 5'- GAC ATC TCA TCT AGG TCA ACT GC -3' for Rb; and forward, 5'-GGA GCG AGA TCC CTC CAA AAT-3' and reverse, 5'-GGC TGT TGT CAT ACT TCT CAT GG3 ' for glyceraldehyde 3-phosphate dehydrogenase (GAPDH). The PCR data were analyzed using the $2^{-D D C t}$ method, and expression was normalized to that of GAPDH.

\section{Western blotting}

Total cellular proteins were extracted using 1\% NP-40 and $1 \mathrm{mM}$ phenylmethylsulfonyl fluoride and were quantified using the bicinchoninic acid assay (Sigma-Aldrich, St. Louis, MO, USA). The proteins were then separated by SDS-PAGE and electroblotted onto polyvinylidene difluoride membranes. After sequential incubations with primary and secondary antibodies, the bands were visualized by enhanced chemiluminescence using the Immobilon ${ }^{\mathrm{TM}}$ Western Kit (Millipore, Burlington, MA, USA). GAPDH served as a loading control.

\section{Cell proliferation assay}

The proliferation of the GC cell lines SGC-7901 and MKN-28 was assessed using the Cell Counting Kit-8 (CCK8: Beyotime Biotechnology, Shanghai, China). All procedures were performed according to the manufacturer's instructions.

\section{Cell migration and invasion assays}


After $72 \mathrm{~h}$, the cells were harvested following treatment with the Rb-targeting saRNA, dsControl, mock transfection, or saRNA/short-hairpin RNA combinations. Cell invasion and migration were measured via Transwell assays using chambers with membranes containing $8 \mu \mathrm{m}$ pores (Corning, Corning, NY, USA). The cells were suspended in $100 \mu \mathrm{L}$ serum-free medium and seeded in the upper chamber, and the lower chamber was filled with $500 \mu \mathrm{L}$ complete media. After incubation at $37^{\circ} \mathrm{C}$ for $24 \mathrm{~h}$, the Transwell membranes were fixed and stained with $0.5 \%$ Crystal Violet/methanol solution for $30 \mathrm{~min}$. The cells that did not migrate were removed using cotton swabs, and the membranes were photographed using a digital camera. In the cell invasion assay, the Transwell membranes were coated with Matrigel (BD Biosciences, San Jose, CA, USA), and the cell incubation period was $48 \mathrm{~h}$.

\section{In vivo model of tumor growth}

All animal experiments were approved by the Animal Care and Ethics Committee of Shandong Provincial Hospital affiliated to Shandong University and conducted in the Animal Center of Shandong Provincial Hospital affiliated to Shandong University. Animals were treated in accordance with the Guide for the Care and Use of Laboratory Animals (8th edition, National Academies Press). The Rb-targeting saRNA and dsControl saRNA were transfected into GC cells, and the mock control group consisted of GC cells treated with the transfection reagent only. Nude mice were randomly divided into three groups $(n=6)$ consisting of mice injected with the following: (1) GC cells transfected with the Rb-targeting saRNA (saRNA group), (2) GC cells transfected with the dsControl saRNA (dsControl group), and (3) mock transfected GC cells (mock group). The cells $\left(5 \times 10^{6}\right)$ were subcutaneously injected into the right side of nude mice. After 21 days, the mice were sacrificed, and solid tumors were isolated for further analysis.

\section{Statistical analysis}

All experimental results are expressed as the mean \pm standard deviation, and one-way analysis of variance followed by the Tukey-Kramer test for multiple comparisons were conducted using Prism statistical software (GraphPad, San Diego, CA, USA). Differences with a value of $P<0.05$ were considered statistically significant.

\section{Results}

\section{saRNA transfection efficiency}

First, we determined the transfection efficiency of the saRNAs targeting Rb. According to PCR, of the three saRNAs tested, saRNA-3 significantly increased the expression of Rb in both SGC-7901 and MKN-28 cells $(P<0.01)$. However, there was no significant difference in Rb expression between the control groups $(P>$ 0.05 ) and the cells transfected with saRNA-1 or saRNA-2 (Fig. 1).

\section{Significant activation of Rb expression by saRNA in GC cells}


Next, our date showed that saRNA-3 notably increase the Rb expression levels in mRNA and protein (Fig.2). The Rb mRNA (4.96 folds or 4.53 folds) and protein ( 2.87 folds or 2.64 folds) levels after transfected with saRNA-3 were increased compared with the mock group $(P<0.01)$.

\section{Effective inhibition of in vitro proliferation by saRNA in GC cells}

The CCK-8 assay was used to detect the effect of saRNA-3 on SGC-7901 and M-28 cell proliferation. saRNA-3 significantly inhibited the proliferation of both cell types by day 3 after transfection. In addition, there was no significant difference in proliferation between the mock and dsControl groups (Fig. 3).

\section{Effective inhibition of in vivo proliferation by saRNA in GC cells}

For in vivo validation, we established a GC model by subcutaneously inoculating cells into BALB/c nude mice. saRNA-3 decreased the tumor volume and weight compared with the mock and dsControl groups, consistent with the in vitro results (Fig. 4).

\section{Effective inhibition of migration and invasion by saRNA in GC cells}

saRNA-3 transfection decreased the migratory ability of SGC-701 and MKN-28 cells compared with the control groups $(P<0.01 ;$ Fig. 5). Conversely, there was no significant difference between the dsControl and mock groups $(P>0.05)$. Moreover, the invasion assay showed that saRNA-3-transfected SGC-701 and MKN-28 cells exhibited a lower invasion rate compared with the dsControl and mock groups $(\mathrm{P}<$ 0.01; Fig. 6).

\section{Discussion}

Globally, GC is the third most lethal tumor, with a mortality rate of approximately 723,000 patients per year. Despite advances in medical technologies, the clinical manifestations of GC are not obvious, and it is usually not detected until an advanced stage. Therefore, the mortality of GC patients therefore has remained high. GC is a heterogeneous disease associated with a variety of genetic backgrounds and epigenetic changes, including genetic mutations, somatic cell copy number changes, structural variations, and epigenetic variations. Characterizing the molecular mechanisms involved in GC progression is therefore a difficult challenge. However, identifying new molecular targets in GC may lead to novel approaches to determine disease prognosis and suitable clinical treatment strategies.

Since the discovery of saRNAs, different RNAa models have been developed, and numerous studies have been conducted on this class of molecules. Janowski and Li et al. confirmed that different saRNAs activate E-cadherin, p21, VEGF, and PR and inhibit tumor cell growth $[13,14]$. Junxia et al. reported that saRNAs inhibit breast and bladder cancers by activating E-cadherin [15]. Kang et al. established an orthotopic tumor model in mice and treated bladder cancer cells with relevant saRNA liquid particles to successfully inhibit tumor growth [16]. Ren also confirmed this mouse model using a similar experiment. Furthermore, saRNAs inhibited prostate cancer cell growth and prolonged survival in mice [17]. In our 
previous study, we designed an saRNA targeting the VEZT gene and found it inhibited the proliferation, invasion, and migration of GC cells via upregulated expression of VEZT [18].

Kundson et al. evaluated the genetic basis of childhood retinoblastoma in 1971 and found that tumor formation requires simultaneous deletion of $13 q 14$ or inactivation of a pair of alleles of the Rb gene. Lee et al. sequenced and predicted that the protein corresponded to this RNA, and the gene was identified and isolated in 1989. Because the gene is closely related to the occurrence of retinoblastoma, it was named the $R b$ gene, which was the first successfully cloned tumor suppressor gene [19]. Recently, it was reported that the $\mathrm{Rb}$ gene is closely related to the development of many malignant tumors such as retinoblastomas, osteosarcomas [20], breast cancer [21], bladder cancer [22], and lung cancer [23]. Furthermore, inactivation of $\mathrm{Rb}$ contributes to tumor formation.

In the present study, we first used three saRNAs to reactivate Rb expression in GC cells (SGC-7901 and MKN-28) and selected saRNA-3 because it most effectively upregulated Rb expression. saRNA-3 inhibited $\mathrm{GC}$ cell proliferation both in vivo and in vitro.

\section{Conclusion}

Our findings also confirmed that saRNA-3 significantly inhibited GC cell invasion and migration. These results indicate that saRNA-mediated upregulation of Rb inhibited the tumorigenicity of GC cells. Targeting specific genes via saRNAs may provide new therapeutic options that could significantly advance the treatment of cancer and other diseases. However, additional studies are needed to identify the mechanism of RNAa, the optimal method for designing saRNAs, and the impact of RNAa on other treatments.

\section{Declarations}

\section{Ethics approval and consent to participate}

The present study was approved by the Ethics Committee of Shandong Provincial Hospital affiliated to Shandong University. Written informed consent was obtained from all enrolled subjects.

\section{Consent to publish}

Not applicable.

\section{Availability of data and materials}

The data used to support the findings of this study are included in the article.

\section{Competing Interest}

There are no conflicts of interest to declare. 


\section{Funding}

This work was funded by grants from the Focus on Research and Development Plans in Shandong province (project No.2017GSF221003, No. 2018GSF118100) and a Jinan Clinical Medicine Science and Technology Innovation Plan, China (project No. 201805069).

\section{Authors' contributions}

WGC conceived the study. PLP, ZSJ and KS designed the experiments; TSB, XT and PLP conducted the experiments. PLP, WGC and ZSJ wrote the manuscript. All authors have read and approved the final manuscript.

\section{Abbreviations}

GC: Gastric cancer;

$\mathrm{Rb}$ : Retinoblastoma

saRNAs: Small activating RNAs

RNAa: RNA activation

dsControl: negative control saRNA

\section{References}

1. Liu J, Li Y, Zou Y, Zhang J, An J, Guo J, Ma M, Dai D: MicroRNA-497 acts as a tumor suppressor in gastric cancer and is downregulated by DNA methylation. Oncology reports 2017, 38(1).

2. Fang Y, Wu C, Gu X, Li Z, Xiang J, Chen Z: Perioperative mortality and morbidity prediction using POSSUM, P-POSSUM and APACHE II in Chinese gastric cancer patients: surgical method is a key independent factor affecting prognosis. International Journal of Clinical Oncology 2014, 19(1):74-80.

3. Conteduca V, Sansonno D, Lauletta G, Russi S, Ingravallo G, Dammacco F: H. pylori infection and gastric cancer: state of the art (review). International Journal of Oncology 2013, 42(1):5-18.

4. Li CF, Zheng J, Xue YW: The value of contrastenhanced computed tomography in predicting gastric cancer recurrence and metastasis. Cancer Biomarkers 2017, 19(3):327.

5. Grant Stemmermann M, Heffelfinger P, Sue C., Amy Noffsinger M, Zhong Hui Y, Miller, Mary Ann, Fenoglio-Preiser, Cecilia M.: The molecular biology of esophageal and gastric cancer and their precursors: Oncogenes, tumor suppressor genes, and growth factors. Human Pathology 1994, 25(10):968.

6. Kang MR, Yang G, Place RF, Charisse K, Epstein-Barash H, Manoharan M, Li LC: Intravesical delivery of small activating RNA formulated into lipid nanoparticles inhibits orthotopic bladder tumor growth. Cancer Research 2012, 72(19):5069-5079. 
7. Kai Y, Xiang-Yi Z, Jie Q, Yun-Bin W, Yu B, Qi-Qi M, Qun W, Zhi-Ming W, Li-Ping X: Up-regulation of p21WAF1/Cip1 by saRNA induces G1-phase arrest and apoptosis in T24 human bladder cancer cells. Cancer Letters 2008, 265(2):206-214.

8. Turner M, Jiao A, Slack FJ: Autoregulation of lin-4 microRNA transcription by RNA activation (RNAa) in C. elegans. Cell Cycle 2014, 13(5):772-781.

9. Mao Q, Zheng X, Yang K, Qin J, Bai Y, Jia X, Li Y, Xie L: Suppression of migration and invasion of PC3 prostate cancer cell line via activating E-cadherin expression by small activating RNA. Cancer Investigation 2010, 28(10):1013-1018.

10. Pushparaj PN, Aarthi JJ, Kumar SD, Manikandan J: RNAi and RNAa-the yin and yang of RNAome. Bioinformation 2008, 2(6):235-237.

11. Kosaka M, Kang MR, Yang G, Li LC: Targeted p21WAF1/CIP1 activation by RNAa inhibits hepatocellular carcinoma cells. Nucleic Acid Therapeutics 2012, 22(5):335-343.

12. Portnoy V, Lin SHS, Li KH, Burlingame A, Hu ZH, Li H, Li LC: saRNA-guided Ago2 targets the RITA complex to promoters to stimulate transcription. Cell Research 2016, 26(3):320-335.

13. Li LC, Okino ST, Zhao H, Pookot D, Place RF, Urakami S, Enokida H, Dahiya R: Small dsRNAs induce transcriptional activation in human cells. Proc Natl Acad Sci U S A 2006, 103(46):17337-17342.

14. Janowski BA, Younger ST, Hardy DB, Ram R, Huffman KE, Corey DR: Activating gene expression in mammalian cells with promoter-targeted duplex RNAs. Nature chemical biology 2007, 3(3):166-173.

15. Junxia W, Ping G, Yuan H, Lijun Z, Jihong R, Fang L, Min L, Xi W, Ting H, Ke D et al: Double strand RNA-guided endogeneous E-cadherin up-regulation induces the apoptosis and inhibits proliferation of breast carcinoma cells in vitro and in vivo. Cancer science 2010, 101(8):1790-1796.

16. Kang MR, Yang G, Place RF, Charisse K, Epstein-Barash H, Manoharan M, Li LC: Intravesical delivery of small activating RNA formulated into lipid nanoparticles inhibits orthotopic bladder tumor growth. Cancer Res 2012, 72(19):5069-5079.

17. Ren S, Kang MR, Wang J, Huang V, Place RF, Sun Y, Li LC: Targeted induction of endogenous NKX3-1 by small activating RNA inhibits prostate tumor growth. The Prostate 2013, 73(14):1591-1601.

18. Xie D, Shang L, Peng L, Li L: Up-regulation of VEZT by small activating RNA inhibits the proliferation, invasion and migration of gastric cancer cells. Biochemical and biophysical research communications 2017, 482(4):542-548.

19. Lee WH, Bookstein R, Hong F, Young LJ, Shew JY, Lee EY: Human retinoblastoma susceptibility gene: cloning, identification, and sequence. Science 1987, 235(4794):1394-1399.

20. Gao YF, Zhang QJ, Yu Z, Liu SH, Liang J: miR-142 suppresses proliferation and induces apoptosis of osteosarcoma cells by upregulating Rb. Oncol Lett 2018, 16(1):733-740.

21. Lin Z, Zhang X, Zhao F, Ru S: Bisphenol S promotes the cell cycle progression and cell proliferation through ERalpha-cyclin D-CDK4/6-pRb pathway in MCF-7 breast cancer cells. Toxicology and applied pharmacology 2019, 366:75-82. 
22. Miyamoto H, Shuin T, Torigoe S, Iwasaki Y, Kubota Y: Retinoblastoma gene mutations in primary human bladder cancer. British Journal of Cancer 1995, 71(4):831-835.

23. Choudhury AD, Beltran H: Retinoblastoma loss in cancer: casting a wider net. Clinical cancer research : an official journal of the American Association for Cancer Research 2019.

\section{Figures}

\section{Figure.1}

A

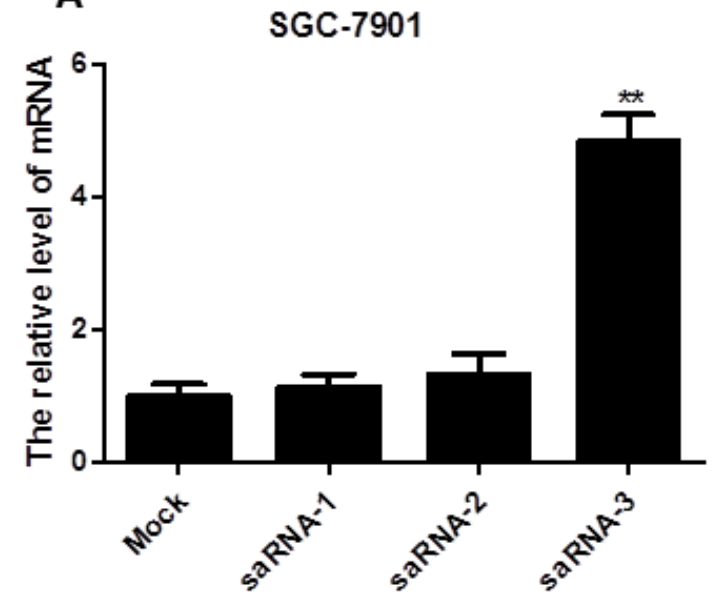

B

SGC-7901

MOCK saRNA-1 saRNA-2 saRNA-3

$\mathbf{R b}$

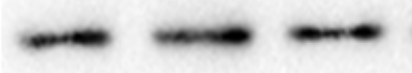

GAPDH

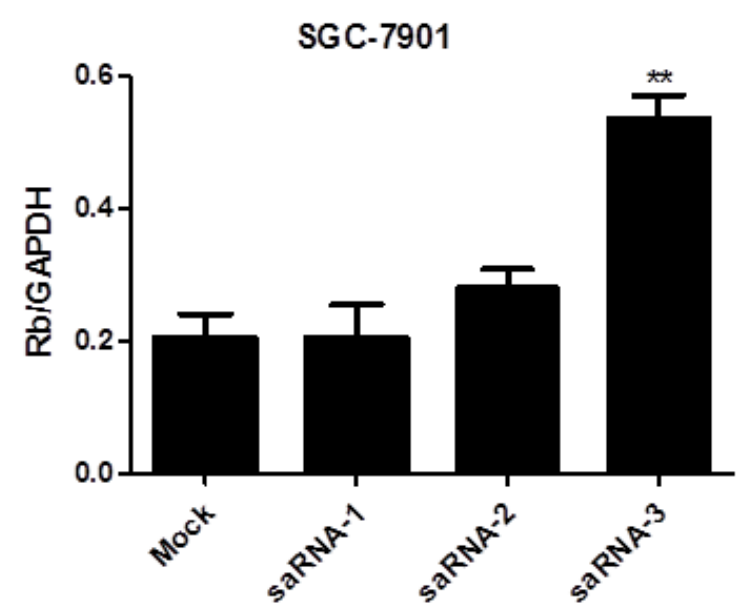

MKN-28

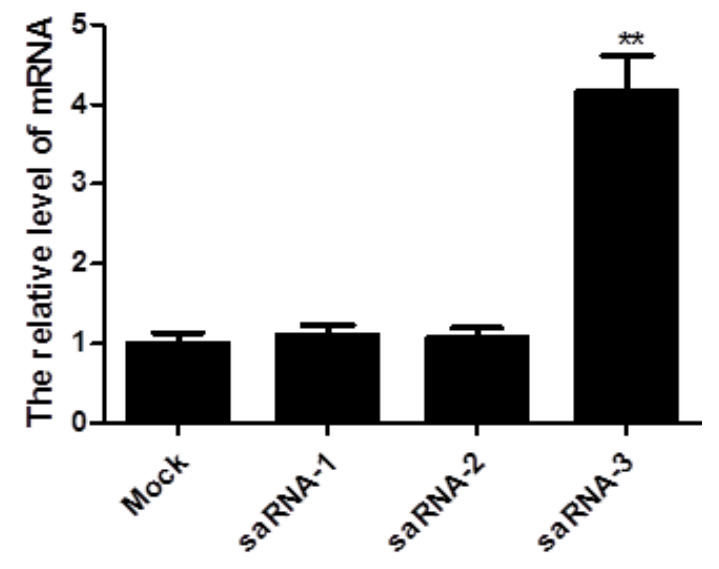

MKN-28

MOCK saRNA-1 saRNA-2 saRNA-3

$\mathbf{R b}$

GAPDH

MKN-28

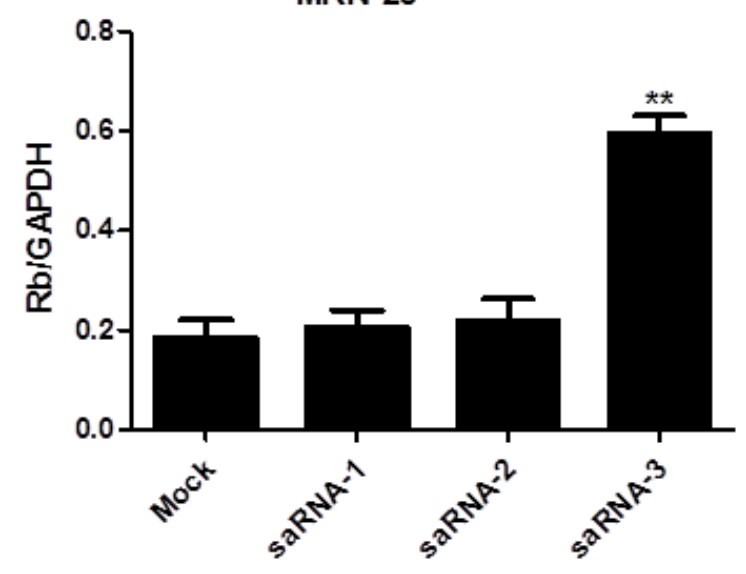


Figure 1

Different saRNAs reactivated the retinoblastoma $(\mathrm{Rb})$ gene with different efficacies. SGC-7901 and MKN28 cells were treated with $50 \mathrm{nM}$ saRNA for $72 \mathrm{~h}$. (A) The Rb mRNA level was assessed by real-time PCR. (B) The Rb protein level was assessed by western blotting. ${ }^{*} P<0.05$, ${ }^{\star *} \mathrm{P}<0.01$ compared with the mock control.

Figure.2
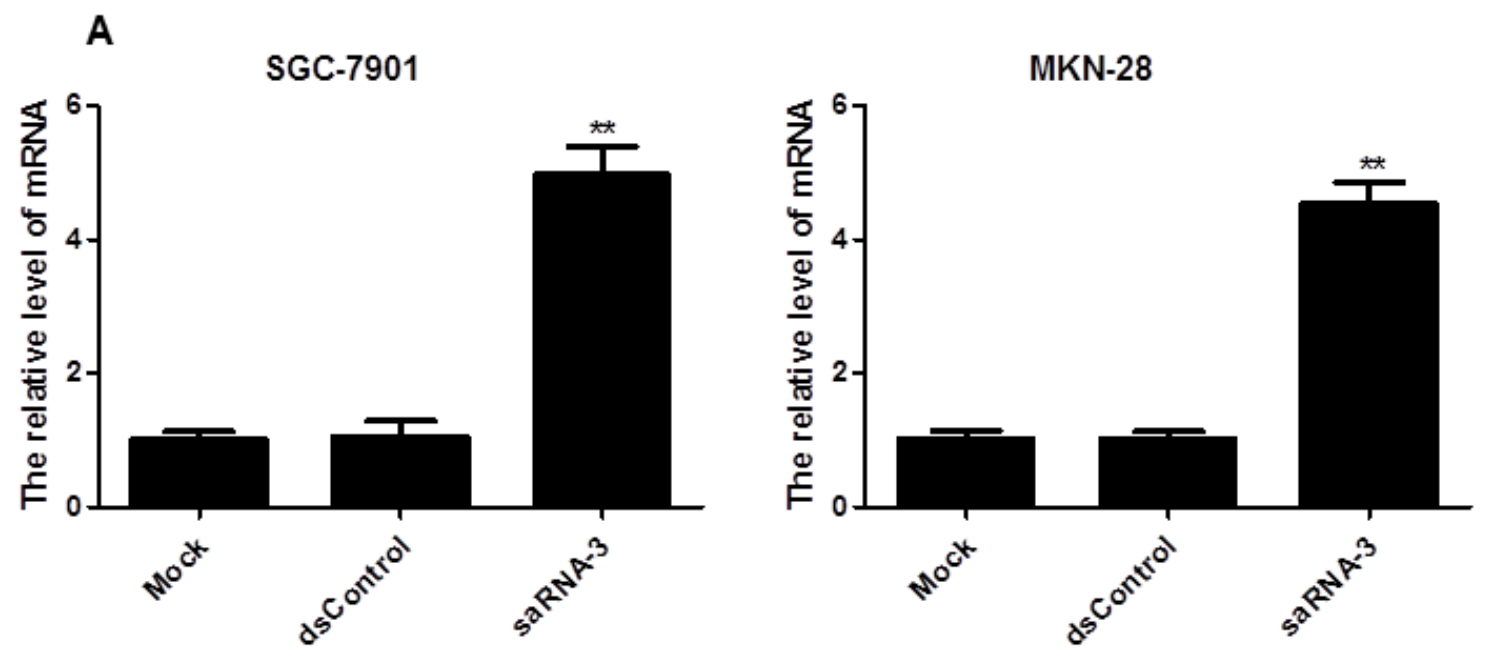

B

SGC-7901

MOCK dsControl saRNA-3

MKN-28

MOCK dsControl saRNA-3

$\mathbf{R b}$

$\mathbf{R b}$

GAPDH

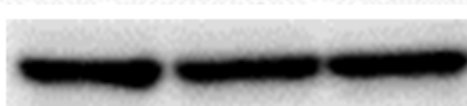

GAPDH
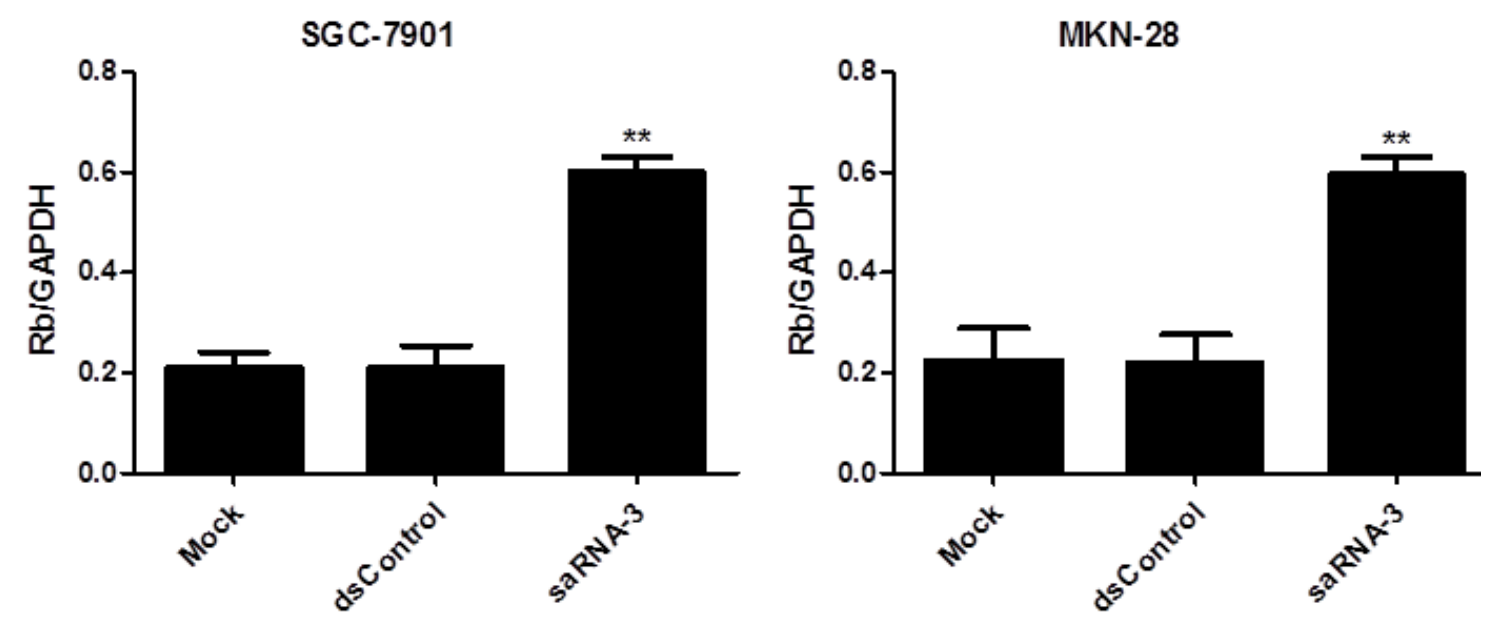

Figure 2 
saRNA-3 induced upregulation of retinoblastoma (Rb) levels. SGC-7901 and MKN-28 cells were treated as described above. The levels of Rb mRNA (A) and protein (B) were detected by real-time PCR and western blot analysis, respectively. ${ }^{*}<0.05,{ }^{*} \mathrm{P}<0.01$ compared with the mock control.

Figure.3

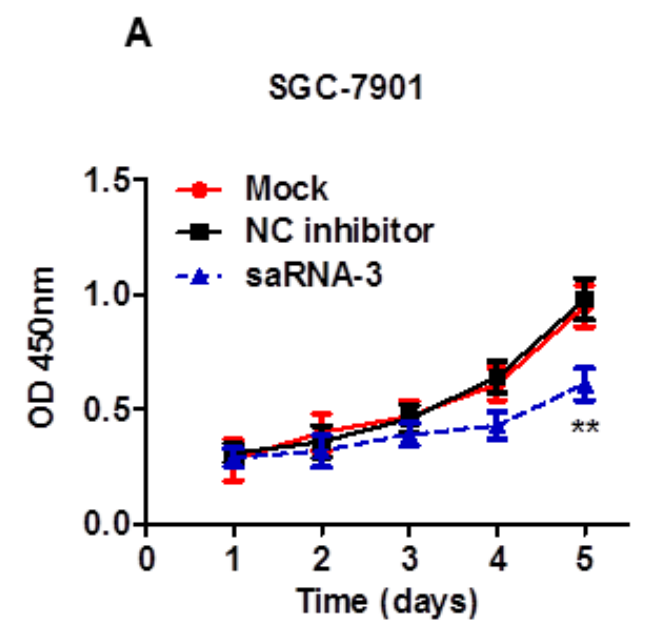

MKN-28

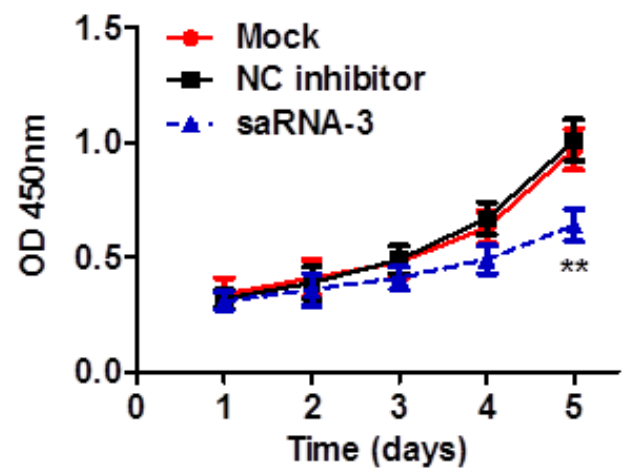

B

SGC-7901

MKN-28
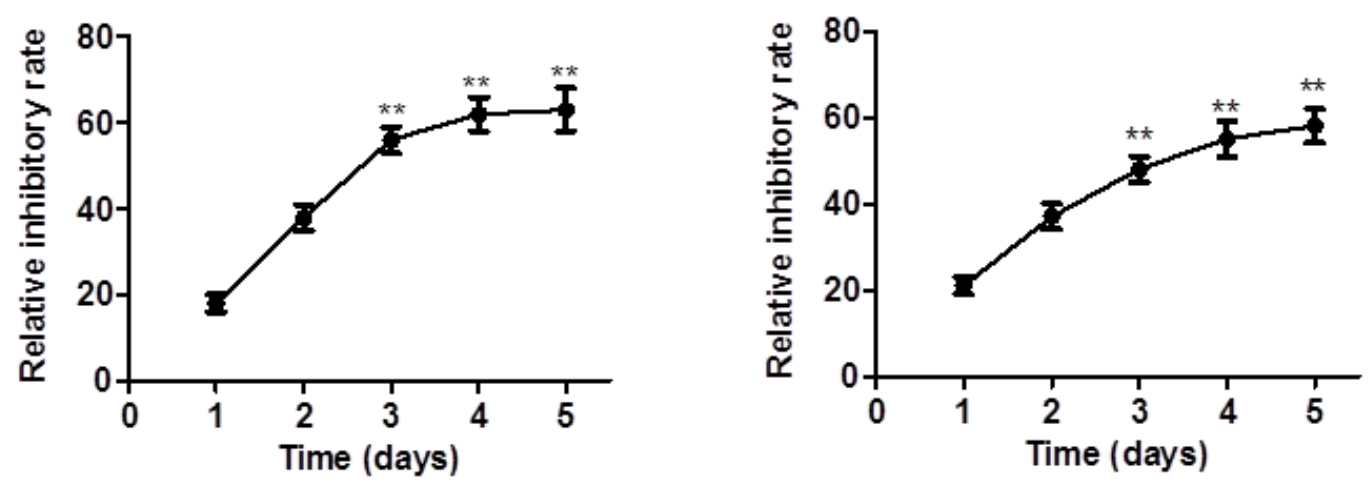

Figure 3

saRNA-3 inhibited the proliferation of gastric cancer cells in vitro. SGC-7901 and MKN-28 cells were treated as described above. Cell proliferation was detected using the CCK-8 assay. saRNA-3 significantly 
inhibited the proliferation of SGC-7901 and MKN-28 cells by day 3 of treatment (A). There was no significant difference between the two control groups (B). ${ }^{*} \mathrm{P}<0.05$, ${ }^{\star *} \mathrm{P}<0.01$ compared with the mock control.

Figure.4

A

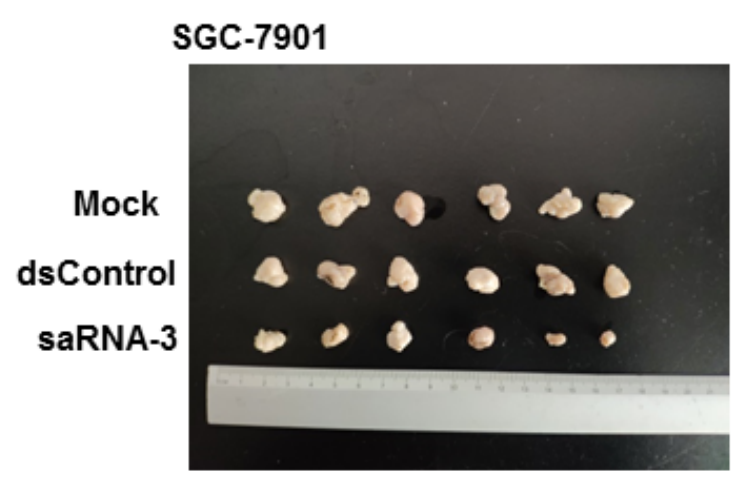

B

SGC-7901
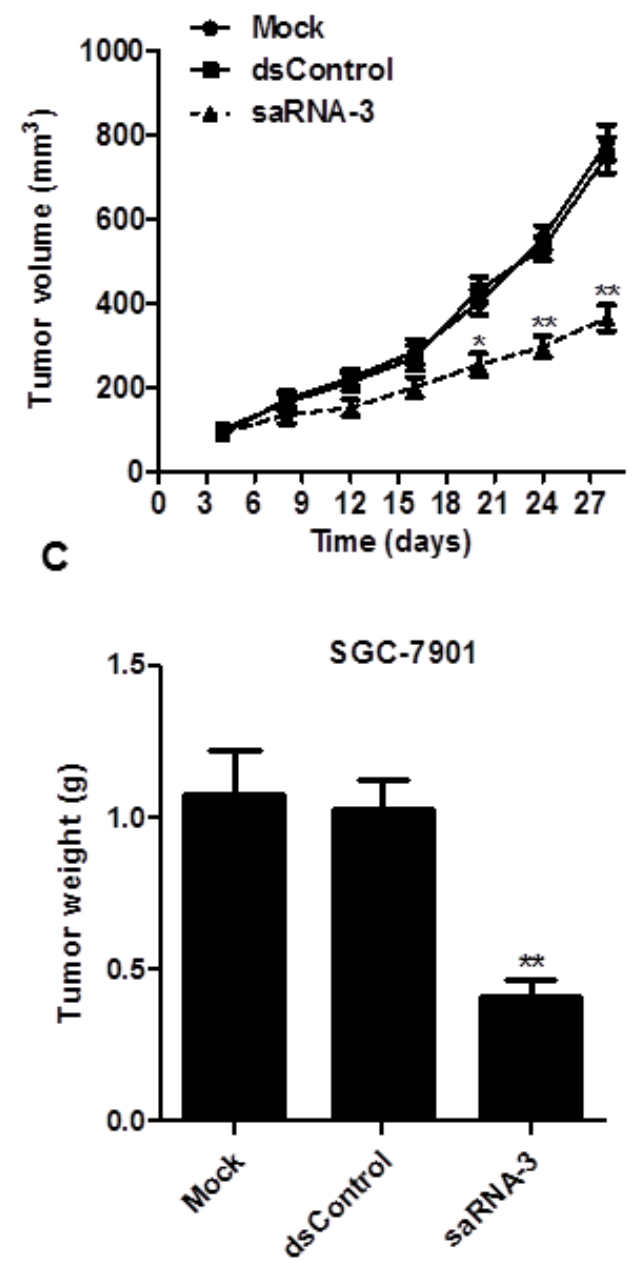

MKN-8

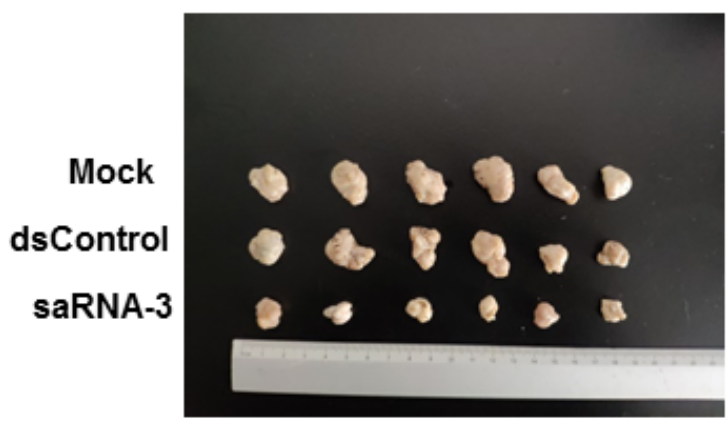

MKN-28
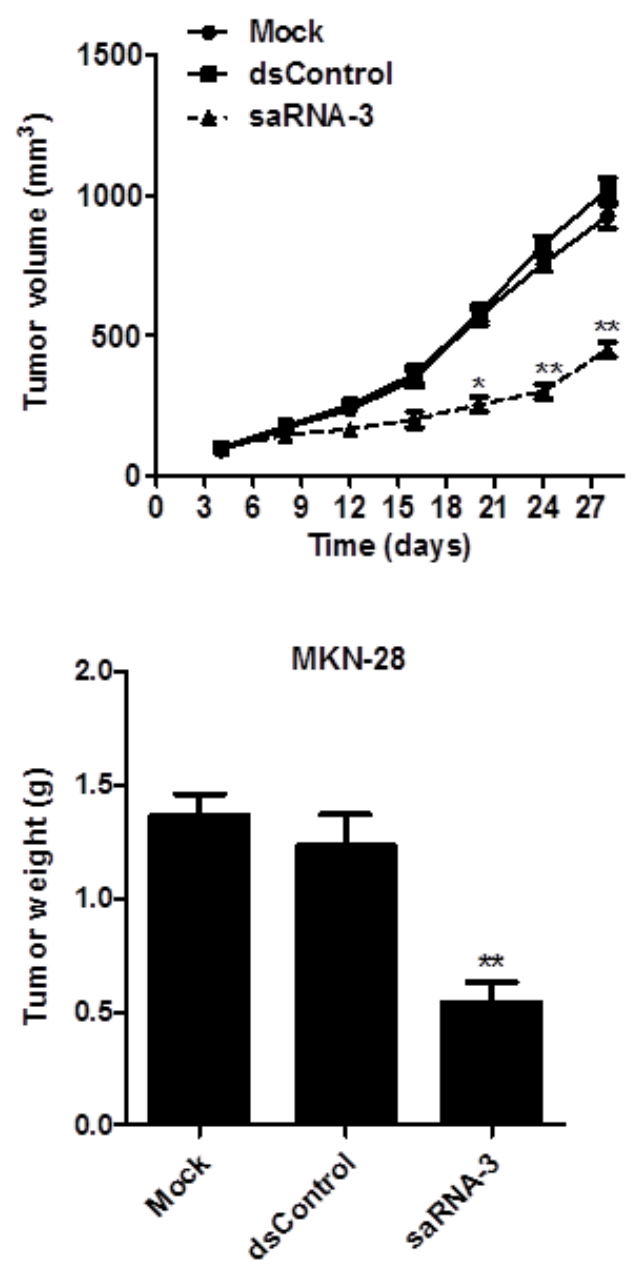

Figure 4

saRNA-3 suppressed tumor growth of gastric cancer cells in vivo. SGC-7901 and MKN-28 cells were treated as described above. Tumor tissue images (A), tumor growth curves (B), and the weight of tumor 
tissues at harvest $(C) .{ }^{*} P<0.05,{ }^{*} \mathrm{P}<0.01$ compared with the mock control.

\section{Figure.5}

\section{A SGC-7901: migration}
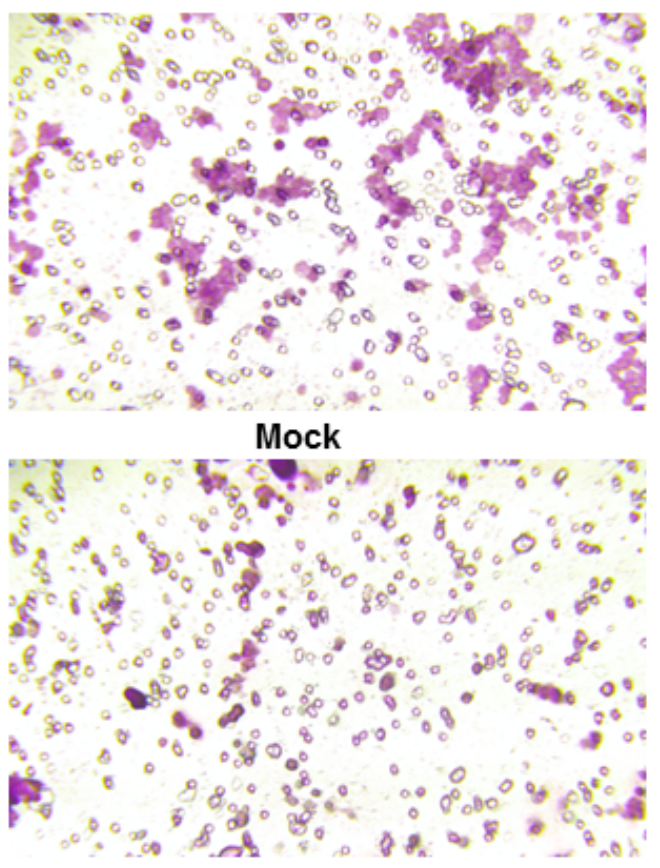

\section{saRNA-3}

\section{B $\quad$ MKN-28: migration}

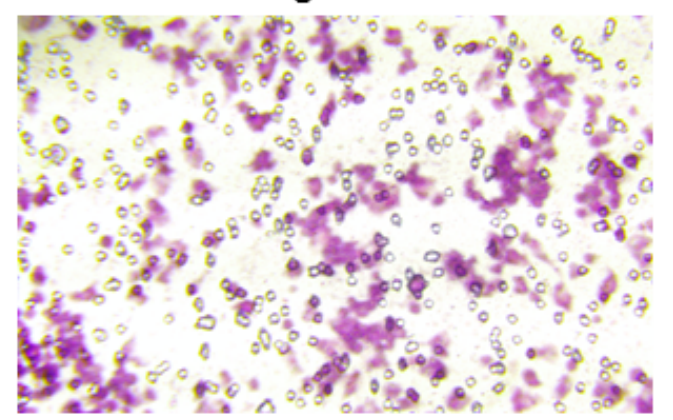

\section{Mock}
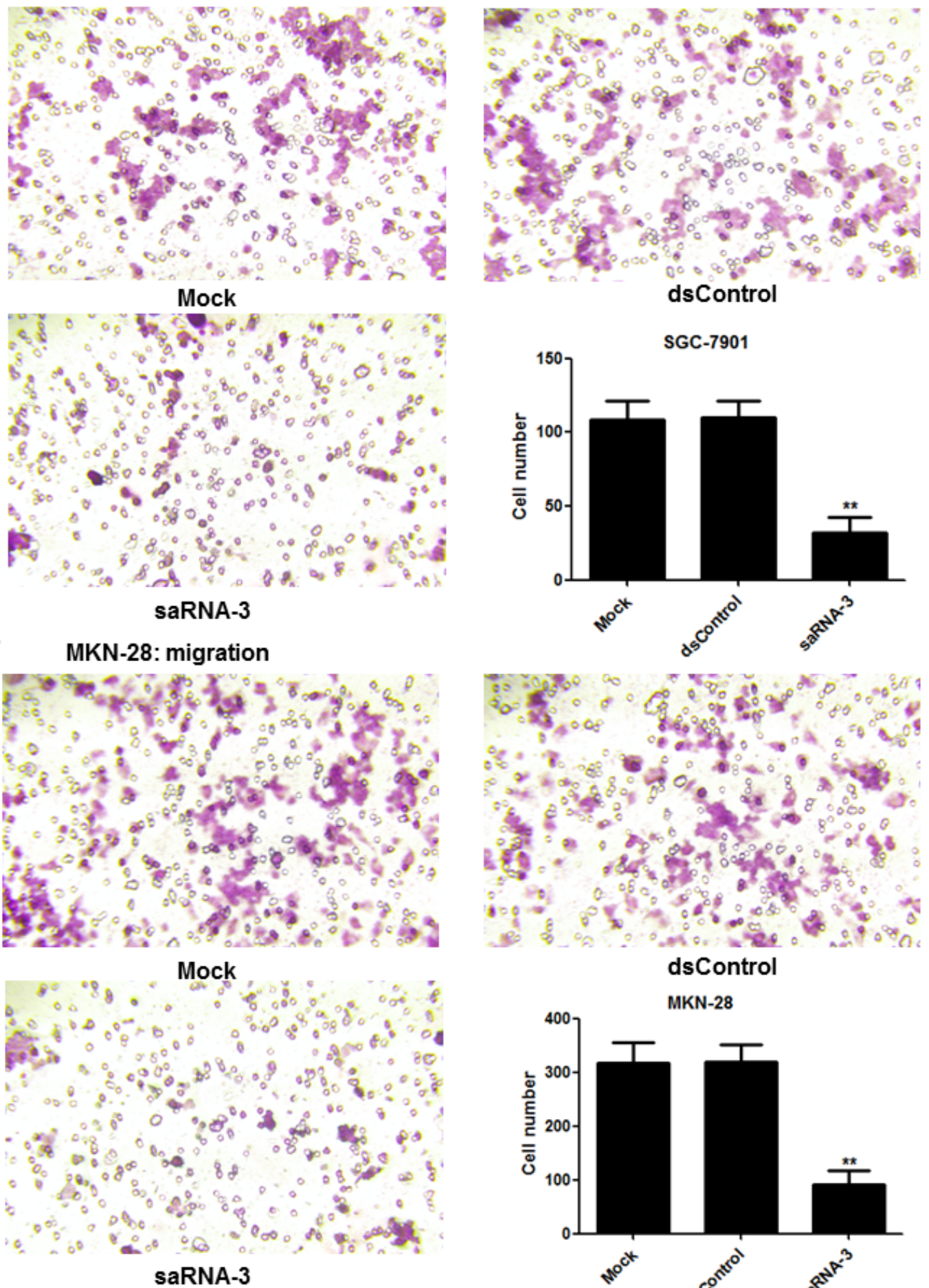

dsControl

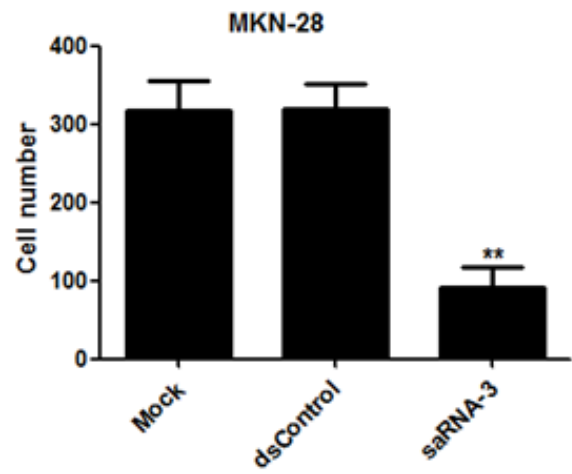

Figure 5

saRNA-3 inhibited the migration of gastric cancer cells. The numbers of migrating SGC-7901 (A) and MKN-28 (B) cells were significantly reduced after saRNA-3 transfection. ${ }^{*} \mathrm{P}<0.05, * \star \mathrm{P}<0.01$ compared with the mock control. 
Figure.6

A SGC-7901: invasion

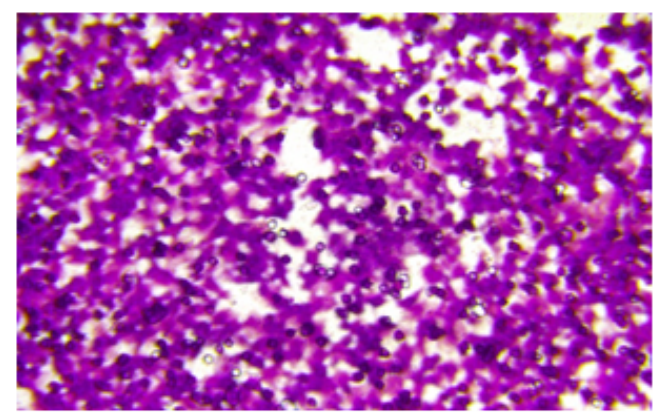
Mock

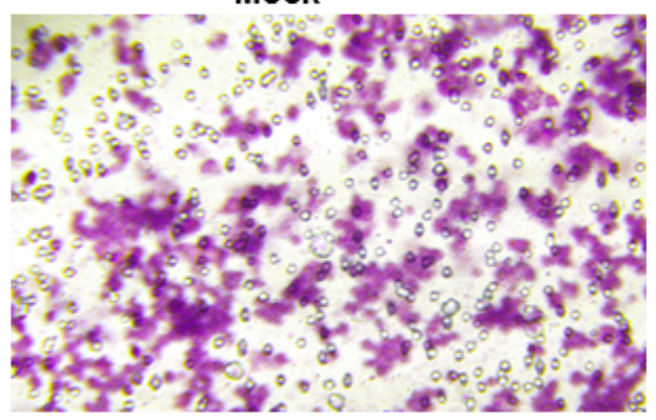

saRNA-3

B

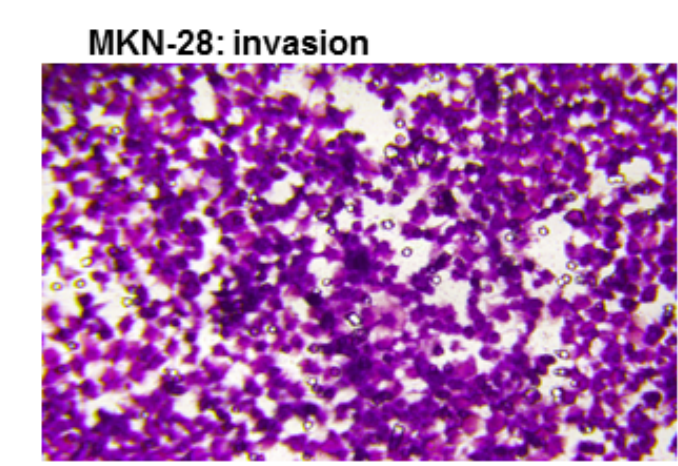

Mock

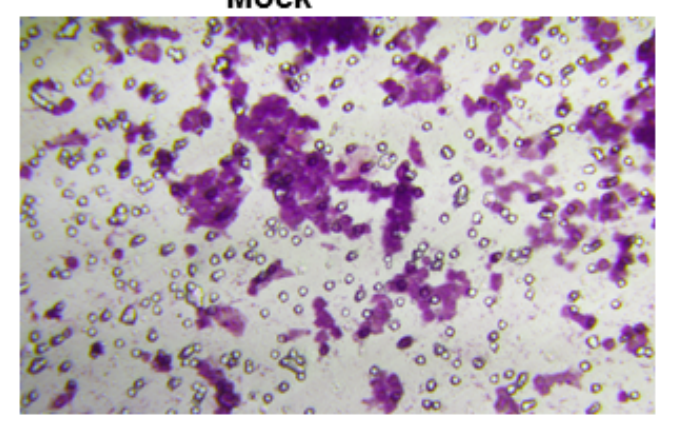

saRNA-3

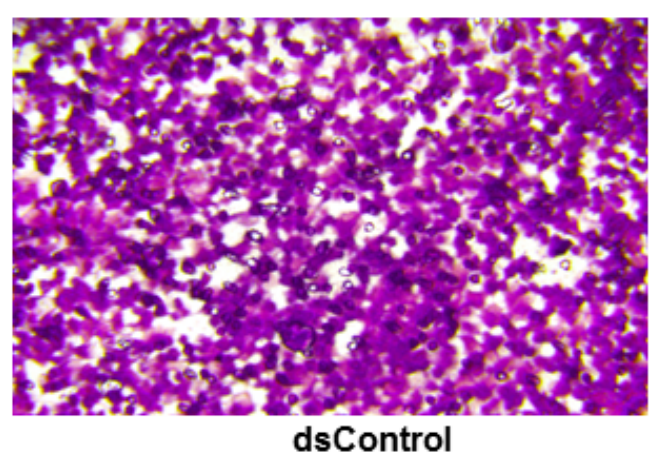

dsControl
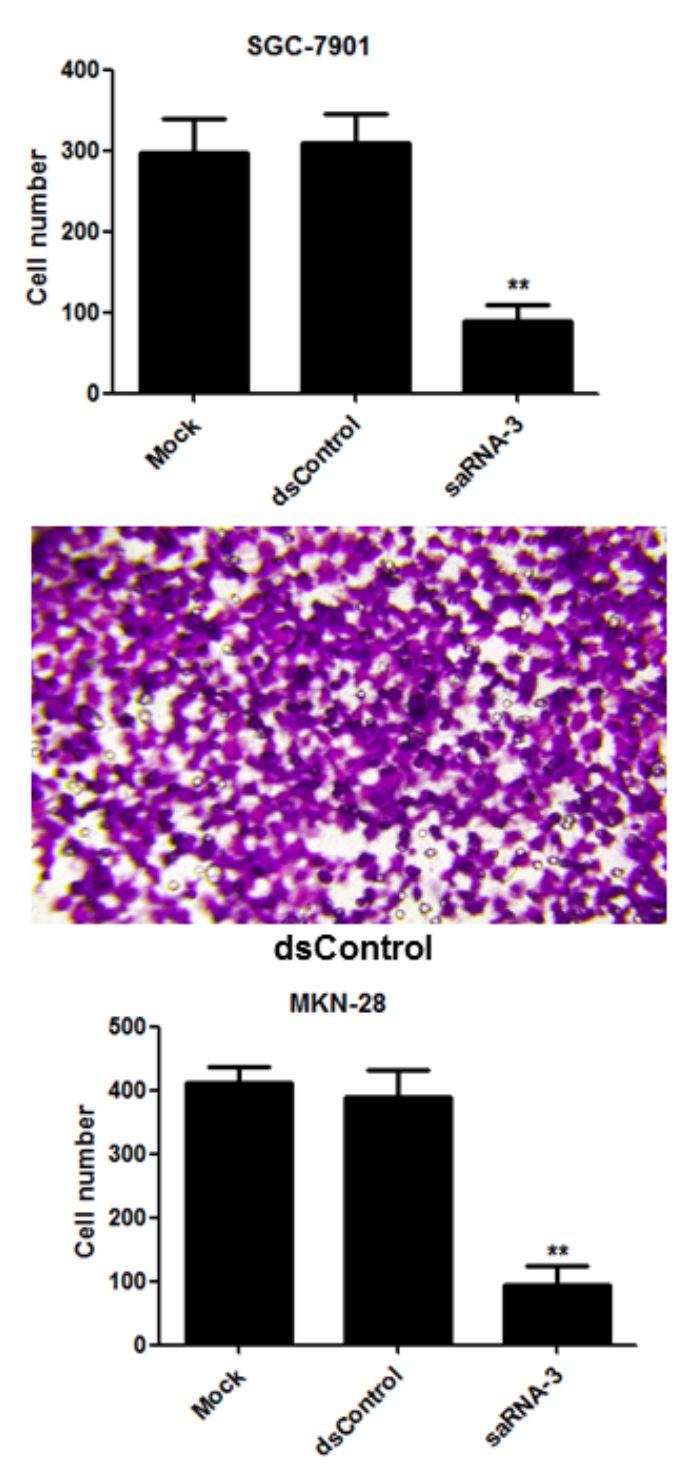

Figure 6

saRNA-3 inhibited the invasion of gastric cancer cells. The numbers of invading SGC-7901 (A) and MKN28 (B) cells were significantly reduced after saRNA-3 transfection. ${ }^{*} P<0.05$, ${ }^{\star} \mathrm{P}<0.01$ compared with the mock control. 